\title{
Dirac-like approach for consistent discretizations of classical constrained theories
}

\author{
Cayetano Di Bartolo ${ }^{1}$, Rodolfo Gambini ${ }^{2}$, Rafael A. Porto ${ }^{3}$ and Jorge Pullin ${ }^{4}$ \\ 1. Departamento de Física, Universidad Simón Bolívar, \\ Aptdo. 89000, Caracas 1080-A, Venezuela. \\ 2. Instituto de Física, Facultad de Ciencias, \\ Iguá 4225, esq. Mataojo, Montevideo, Uruguay. \\ 3. Department of Physics, \\ Carnegie Mellon University, Pittsburgh, PA 15213 \\ 4. Department of Physics and Astronomy, \\ Louisiana State University, \\ Baton Rouge, LA 70803-4001
}

\begin{abstract}
We analyze the canonical treatment of classical constrained mechanical systems formulated with a discrete time. We prove that under very general conditions, it is possible to introduce nonsingular canonical transformations that preserve the constraint surface and the Poisson or Dirac bracket structure. The conditions for the preservation of the constraints are more stringent than in the continuous case and as a consequence some of the continuum constraints become second class upon discretization and need to be solved by fixing their associated Lagrange multipliers. The gauge invariance of the discrete theory is encoded in a set of arbitrary functions that appear in the generating function of the evolution equations. The resulting scheme is general enough to accommodate the treatment of field theories on the lattice. This paper attempts to clarify and put on sounder footing a discretization technique that has already been used to treat a variety of systems, including Yang-Mills theories, BF-theory and general relativity on the lattice.
\end{abstract}

\section{INTRODUCTION}

We have recently introduced [1, 2] a technique for treating the theories that arise when one discretizes (space)time in a constrained mechanical system or a continuum field theory. We have shown that this technique works for Yang-Mills and BF theories and implemented it for the gravitational case. Previous attempts to studying systems with discrete time had concentrated mostly on systems without constraints or with holonomic constraints only (for a review with a comprehensive reference list see [3].)

The idea consists on starting from a discretized action, constructing discrete Lagrange equations and introducing a symplectic structure in the discrete space. The evolution is implemented via canonical transformations and the consistency of the discrete theory determines in part the Lagrange multipliers. In some totally constrained systems, like general relativity, the resulting discrete theories are constraint-free since the constraints are solved for the Lagrange multipliers. This makes the quantization of the discrete theories considerably simpler than the continuum cases. This was exploited to make progress in solving the problem of time in quantum gravity [4, 5] and to implement the PageWootters relational time [5, 6] and show that a fundamental decoherence arises in quantum mechanics from quantum gravity.

In this paper, we want to address in a more systematic way the issue of the canonical formulation of discrete constrained systems. Up to now, most of the analysis has been made on specific examples, and a canonical analysis, a la Dirac [7, [8], is still lacking. In particular, the technique relied heavily on defining a canonical transformation that was initially singular (and therefore not a true canonical transformation) and showing that one could eliminate variables and end up with a true canonical transformation. Up to now this was shown in a case by case basis. This paper determines the general conditions needed for the construction of a proper canonical transformation by following a close analogue of the Dirac procedure, adapted to the discrete case. In particular, we note that there are several ways to proceed that yield equivalent results, but that may offer different advantages for particular systems.

In section II we will lay out the framework of how to deal with mechanical systems where time is discrete, including singular and non-singular systems. In section III we will develop a classification of constraints into first and second class suitable for the discrete context. In section IV we work out a specific example that exhibits the details of the formalism. In section $\mathrm{V}$ we discuss an alternative formulation of the formalism and we end with conclusions and discussion.

\section{MECHANICS WITH DISCRETE TIME}

We start by considering a continuum theory representing a mechanical system. Its Lagrangian will be denoted by $\hat{L}\left(q^{a}, \dot{q}^{a}\right), a=1 \ldots M$. This setting is general enough to accommodate, for instance, totally constrained systems. In 
such case $\dot{q}$ will be the derivative of the canonical variables with respect to the evolution parameter. It is also general enough to include the systems that result from formulating on a discrete space-time lattice a continuum field theory.

We discretize the evolution parameter in intervals (possibly varying upon evolution) $t_{n+1}-t_{n}=\epsilon_{n}$ and we label the generalized coordinates evaluated at $t_{n}$ as $q_{n}$. We define the discretized Lagrangian as

$$
L(n, n+1) \equiv L\left(q_{n}^{a}, q_{n+1}^{a}\right) \equiv \epsilon_{n} \hat{L}\left(q^{a}, \dot{q}^{a}\right)
$$

where

$$
q=q_{n} \quad \text { and } \quad \dot{q} \equiv \frac{q_{n+1}-q_{n}}{\epsilon_{n}}
$$

The action can then be written as

$$
S=\sum_{n=1}^{N} L(n, n+1)
$$

If the continuum theory is invariant under reparameterizations of the evolution parameter, one can show that the information about the intervals $\epsilon_{n}$ may be absorbed in the Lagrange multipliers. In the case of standard mechanical systems it is simpler to use an invariant interval $\epsilon_{n}=\epsilon$.

The Lagrange equations of motion are obtained by requiring the action to be stationary under variations of the configuration variables $q^{a}$ fixed at the endpoints of the evolution interval $n=0, n=N+1$,

$$
\frac{\partial L(n, n+1)}{\partial q_{n}^{a}}+\frac{\partial L(n-1, n)}{\partial q_{n}^{a}}=0 .
$$

These equations define a unique evolution if the determinant

$$
\left|\frac{\partial^{2} L(n, n+1)}{\partial q_{n+1}^{b} \partial q_{n}^{a}}\right| \neq 0
$$

We will refer to this case as the nonsingular case. When the determinant vanishes, one will have to analyze the situation differently. Let us start with the nonsingular case.

\section{A. Nonsingular case}

In this case one can solve the Lagrange equations explicitly and the $q_{n+1}$ are uniquely given as a function of $q_{n}$ and $q_{n-1}$. This is the equivalent of the Hessian condition for the non-singular Lagrangian theories in the continuum. The resulting equations are "second order" in the sense that the $q_{n}$ 's are determined provided one knows two previous time levels. One can introduce a "first order" formulation by introducing canonically conjugate variables as is usually done when introducing a Hamiltonian formulation in the continuum theories.

We introduce the following definition of variables that we will later show end up being canonically conjugate momenta of the configuration variables,

$$
\begin{aligned}
p_{n+1}^{a} & \equiv \frac{\partial L(n, n+1)}{\partial q_{n+1}^{a}} \\
p_{n}^{a} & \equiv \frac{\partial L(n-1, n)}{\partial q_{n}^{a}}=-\frac{\partial L(n, n+1)}{\partial q_{n}^{a}}
\end{aligned}
$$

Where we have used Eq. (4). The equations (6) and (7) define a canonical transformation for the variables $q_{n}, p_{n}$ to $q_{n+1}, p_{n+1}$ with a the type 1 generating function $F_{1}=-L\left(q_{n}^{a}, q_{n}^{a}+1\right)$ provided that condition (5) is fulfilled. Notice that the evolution scheme is implicit, one can use the bottom equation (since we are in the non-singular case) to give an expression for $q_{n+1}$ in terms of $q_{n}, p_{n}$, which in turn can be substituted in the top equation to get an equation for $p_{n+1}$ purely in terms of $q_{n}, p_{n}$.

It should be noted that there are several other possible choices, when going from the set of equations (6 7) to an explicit evolution scheme. For example, one can choose to do things in a way that yields a closer analogy with the 
standard Hamiltonian description in the continuum by introducing type two canonical transformations. To do this, we choose to invert equation (6) for $q_{n+1}^{a} \equiv q_{n+1}^{a}\left(q_{n}^{b}, p_{n+1}^{b}\right)$, which is possible only if

$$
\left|\frac{\partial^{2} L(n, n+1)}{\partial q_{n+1}^{a} \partial q_{n+1}^{b}}\right| \neq 0 \text {. }
$$

We can now introduce a Legendre transform and define

$$
F_{2}\left(q_{n}, p_{n+1}\right) \equiv \sum_{a} q_{n+1}^{a} p_{n+1}^{a}-L\left(q_{n}, q_{n+1}\right) .
$$

From here it is immediate to obtain

$$
\begin{aligned}
q_{n+1}^{b} & =\frac{\partial F_{2}}{\partial p_{n+1}^{b}} \\
\frac{\partial F_{2}}{\partial q_{n}^{b}} & =\frac{-\partial L(n, n+1)}{\partial q_{n}^{b}}=p_{n}^{b}
\end{aligned}
$$

where we have used the Lagrange equation in order to obtain the last equality. We easily recognize from here that $F_{2}$ behaves as a type two generating function of the canonical transformation connecting level $n$ with level $n+1$. by,

We may define now a sort of "type 2 " Hamiltonian (in the sense that it depends on $p_{n+1}$ and $q_{n}, H_{2}\left(p_{n+1}, q_{n}\right)$ given

$$
H_{2}\left(q_{n}, p_{n+1}\right) \equiv \sum_{a} p_{n+1}^{a}\left(q_{n+1}^{a}-q_{n}^{a}\right)-L\left(q_{n}, q_{n+1}\right)=F_{2}\left(q_{n}, p_{n+1}\right)-\sum_{a} p_{n+1}^{a} q_{n}^{a} .
$$

which leads to the discrete Hamilton equations,

$$
\begin{aligned}
q_{n+1}^{b} & =q_{n}^{b}+\frac{\partial H_{2}}{\partial p_{n+1}^{b}} \\
p_{n}^{b} & =p_{n+1}^{b}+\frac{\partial H_{2}}{\partial q_{n}^{b}} .
\end{aligned}
$$

It should be noted that although this formulation has a degree of analogy with the traditional Hamiltonian formulation, there are significant differences due to the fact that the conjugate variables live at different time slices. It would not be possible therefore to use this formulation to attempt to construct a Schrödinger equation starting from the above Hamiltonian.

Provided that the canonical map defined by $F_{2}$ is invertible we end up with a discrete evolution implemented by a canonical transformation. It can be easily seen by using the Legendre transform that $F_{2}$ will be invertible if and only if

$$
\left|\frac{\partial^{2} F_{2}(n, n+1)}{\partial q_{n}^{a} p_{n+1}^{b}}\right|=-\left|\frac{\partial^{2} L(n, n+1)}{\partial q_{n}^{a} q_{n+1}^{b}}\right| \times\left|\frac{\partial^{2} L(n, n+1)}{\partial q_{n+1}^{a} q_{n+1}^{b}}\right|^{-1} \neq 0
$$

Thus, in order to have canonical transformations generated by type two functions, the Hessian condition in the continuum time mechanics leads to two independent conditions in the discrete theory given by Eqs. (5) and (8). Notice however that (8) is not necessary for introducing a symplectic structure.

It is clear that when one builds a canonical discrete theory there are four possibilities depending on which pair of variables one chooses to construct the generating functional of the canonical transformation, either $q_{n}, q_{n+1}, q_{n}, p_{n+1}$, $p_{n}, q_{n+1}, p_{n}, p_{n+1}$. In this subsection we considered only two, but the others can be easily generalized from the discussion here.

\section{B. The singular case}

Let us consider as before the generic discrete Lagrangian $L(n, n+1)=L\left(q_{n}^{a}, q_{n+1}^{a}\right)$ with $a=1 \ldots M$. It leads to the equations we already discussed,

$$
\begin{aligned}
p_{n+1}^{a} & =\frac{\partial L(n, n+1)}{\partial q_{n+1}^{a}} \\
p_{n}^{a} & =-\frac{\partial L(n, n+1)}{\partial q_{n}^{a}}
\end{aligned}
$$


If $\left|\frac{\partial^{2} L(n, n+1)}{\partial q_{n+1}^{a} \partial q_{n}^{b}}\right|$ vanishes and its rank is $K$, the system is singular and has $M-K$ constraints of the form

$$
\Phi_{A}\left(q_{n}^{a}, p_{n}^{a}\right)=0
$$

that result from Eq[17], and $M-K$ constraints

$$
\Psi_{A}\left(q_{n+1}^{a}, p_{n+1}^{a}\right)=0
$$

resulting from $\mathrm{Eq(16)}$. The evolution of the configuration variables from level $n$ to $n+1$ is given by solving for $q_{n+1}^{a}$ the Eqs. (17). As the system is singular the evolution depends of $(M-K)$ arbitrary functions $V^{A}$

$$
q_{n+1}^{a}=f^{a}\left(q_{n}^{b}, p_{n}^{b}, V^{A}\right)
$$

We shall follow closely the standard Dirac canonical procedure of continuum mechanics. In this case, the analysis of a constraint system goes trough two steps. The first step consists in the definition of a set of evolution equations that weakly preserve the constraints and the Poisson symplectic structure. To do that one defines the total Hamiltonian $H_{T}=H_{0}+V^{\alpha} \phi_{\alpha}$ where $\phi_{\alpha}$ are the primary constraints, and the $V^{\alpha}$ are partially determined in order to preserve all the constraints of the system. Even though some of the $V^{\prime} s$ may be arbitrary functions, once they are specified, the evolution generated by $H_{T}$ preserves the Poisson brackets and the dynamical evolution is consistent at the classical level with the constraint structure. The second step is only required to quantize the system and consists in the identification of the first and second class constraints and the introduction of the Dirac brackets that enforce strongly the second class constraints.

As we shall see, the same procedure may be followed in the discrete case. The main difference is the implementation of the canonical transformation that is not generated by a Hamiltonian but by a canonical transformation of type 2 , 3 , or 4 .

Let us start by completing the evolution equations. We need to add to Eq(20) an equation for $p_{n+1}$

$$
p_{n+1}^{a}=\left.\frac{\partial L\left(q_{n}, q_{n+1}\right)}{\partial q_{n+1}^{a}}\right|_{q_{n+1}^{a}=f^{a}\left(q_{n}^{b}, p_{n}^{b}, V^{A}\right)} .
$$

We now impose the preservation of the constraints

$$
\Phi_{A}\left(q_{n+1}^{a}, p_{n+1}^{a}\right)=\Phi_{A}\left(f_{n}^{a}, \frac{\partial L\left(q_{n}, f^{a}\right)}{\partial q_{n+1}^{a}}\right)=0
$$

Furthermore, we need to impose the $n+1$ level constraints at level $n, \Psi_{A}\left(q_{n}^{a}, p_{n}^{a}\right)=0$ and impose the consistency conditions

$$
\Psi_{A}\left(f_{n}^{a}, \frac{\partial L\left(q_{n}, f^{a}\right)}{\partial q_{n+1}^{a}}\right)=0
$$

Three different cases may occur:

a) Eqs. 2223) vanish automatically, and therefore we are not led to new conditions.

b) They lead to inconsistencies, and the dynamical system is inconsistent.

c) New secondary constraints $C\left(q_{n}^{a}, p_{n}^{a}\right)$ appear or/and some of the arbitrary functions $V^{A}$ are determined, that is $V^{A}=V^{A}\left(q_{n}^{a}, p_{n}^{a}, v^{\alpha}\right)$ with $\alpha=1 \ldots R \leq(N-K)$, and $v^{\alpha}$ arbitrary functions. The process is repeated until consistency is achieved. That is, until the consistency conditions are automatically satisfied without further constraints and conditions for $V$.

Substituting $V^{A}$ in (20) and (21) we get the evolution equations that preserve all the constraints: primary, secondary, tertiary, and so on,

$$
\begin{gathered}
q_{n+1}^{a}=f^{a}\left(q_{n}^{b}, p_{n}^{b}, V^{A}(q, p, v)\right)=\tilde{f}^{a}\left(q_{n}^{b}, p_{n}^{b}, v^{\alpha}\right) \\
p_{n+1}^{a}=g^{a}\left(q_{n}^{b}, p_{n}^{b}, v^{\alpha}\right)
\end{gathered}
$$


Initial values need to be restricted by the $n=0$ level constraints,

$$
\Phi_{A}\left(q_{0}^{a}, p_{0}^{a}\right)=\Psi_{A}\left(q_{0}^{a}, p_{0}^{a}\right)=0 .
$$

In order to have a complete analogy with the continuum case, we still need to analyze under what conditions this evolution also preserves the Poisson bracket structure. As in the continuum case we assume that the arbitrary functions $v$ have been fixed. Three different cases may arise depending on if one chooses to implement things in terms of a canonical transformation of type 2,3 or 4 :

Case I) The equation(24) is invertible for $q_{n}^{a}$ : that is, $\left|\frac{\partial q_{n+1}^{a}}{\partial q_{n}^{b}}\right| \neq 0$ and therefore one can write

$$
q_{n}^{a}=h^{a}\left(q_{n+1}^{b}, p_{n}^{b}\right) .
$$

Notice that, under these hypotheses, there are no pseudo-constraints of the form $G\left(q_{n+1}^{a}, p_{n}^{a}\right)=0$. We call these pseudo-constraints because they involve variables at different instants of time.

We may define a type 3 generating functions of canonical transformations,

$$
F_{3}\left(q_{n+1}^{b}, p_{n}^{b}\right)=\left.\left[p_{n}^{b} q_{n}^{b}+L\left(q_{n}, q_{n+1}\right)\right]\right|_{q_{n}^{b}=h^{b}} .
$$

Then we have

$$
\frac{\partial F_{3}\left(q_{n+1}^{a}, p_{n}^{a}\right)}{\partial p_{n}^{b}}=q_{n}^{b}=h^{b}\left(q_{n+1}^{a}, p_{n}^{a}\right)
$$

and

$$
\frac{\partial F_{3}\left(q_{n+1}^{a}, p_{n}^{a}\right)}{\partial q_{n+1}^{b}}=\left.\frac{\partial L\left(q_{n}^{a}, q_{n+1}^{a}\right)}{\partial q_{n+1}^{b}}\right|_{q_{n}=h}=p_{n+1}^{b} .
$$

Notice that in the last equality there are also contributions coming from the dependence on $q_{n+1}$ of the level $n$ variables $q_{n}$, but these contributions cancel because of the definition of the canonical momenta. The information about the momenta is completely encoded in the evolution equations $q_{n+1}^{a}=h^{a}$ and the constraints. As the first equation is equivalent to (24) one ends up recovering the fundamental evolution equations as a canonical transformation generated by $F_{3}$. Furthermore,

$$
\left|\frac{\partial F_{3}\left(q_{n+1}^{a}, p_{n}^{a}\right)}{\partial p_{n}^{b} \partial q_{n+1}^{a}}\right|=\left|\frac{\partial q_{n}^{b}}{\partial q_{n+1}^{a}}\right| \neq 0
$$

due to the fact that we have assumed that $\left|\frac{\partial q_{n+1}^{b}}{\partial q_{n}^{a}}\right| \neq 0$, and consequently $F_{3}$ is a non singular generating function and therefore the resulting canonical transformation preserves the Poisson bracket structure.

Case II) The equation(25) is invertible for $p_{n}^{a}$ : that is $\left|\frac{\partial p_{n+1}^{a}}{\partial p_{n}^{b}}\right| \neq 0$ and therefore one can write,

$$
p_{n}^{a}=g^{a}\left(q_{n}^{b}, p_{n+1}^{b}\right)
$$

Notice that, under these hypotheses, there are no pseudo-constraints of the form $G\left(q_{n}^{a}, p_{n+1}^{a}\right)=0$. By substituting (32) in (24), one gets

$$
q_{n+1}^{a}=k^{a}\left(q_{n}^{b}, p_{n+1}^{b}\right),
$$

which allows to introduce a type 2 generating function

$$
F_{2}\left(q_{n}^{b}, p_{n+1}^{b}\right)=\left.\left[p_{n+1}^{b} q_{n+1}^{b}-L\left(q_{n}, q_{n+1}\right)\right]\right|_{q_{n+1}^{b}=k^{b}}
$$

One can now easily check that this generating function reproduces the evolution equations (24) and (25) and defines a non singular canonical transformation that preserves the Poisson Brackets in the evolution.

Case III) Even when the system has pseudo-constraints of the form $G\left(q_{n+1}^{a}, p_{n}^{a}\right)=0$ and $F\left(q_{n}^{a}, p_{n+1}^{a}\right)=0$ one may be able to find a canonical transformation provided that the system does not have pseudo-constraints of the form $F\left(p_{n}^{a}, p_{n+1}^{a}\right)=0$ 
In fact, by using Eq.(25) one can invert for

$$
q_{n}^{a}=l^{a}\left(p_{n}^{b}, p_{n+1}^{b}\right)
$$

and substituting in (24) get

$$
q_{n+1}^{a}=m^{a}\left(p_{n}^{b}, p_{n+1}^{b}\right)
$$

A generating function of type 4 that does the same job that the two previous ones may now be introduced,

$$
F_{4}\left(p_{n}^{b}, p_{n+1}^{b}\right)=\left.\left[-p_{n+1}^{b} q_{n+1}^{b}+p_{n}^{b} q_{n}^{b}+L\left(q_{n}, q_{n+1}\right)\right]\right|_{q_{n}^{b}=l^{b}} ^{q_{n+1}^{b}=m^{b}}
$$

All of the discrete systems that have been treated up to now in the literature may be analyzed by following this canonical procedure, allowing to preserve the constraints and the Poisson bracket structure. Later on we will show an example of a system of this type in order to analyze how this procedure works in a concrete case.

It should be noted that there may exist mechanical systems that do not fall into any of the above classifications. For instance, a system could have pseudo-constraints of all the types listed above. In such cases one will need to develop further techniques to treat them. For instance one could introduce canonical transformations of a given type for some of the variables and of a different type for other variables. This would require further study and it does not appear necessary for the systems that have been analyzed up to present.

\section{CLASSIFICATION OF THE CONSTRAINTS}

At this point we have a set of constraints primary, secondary, tertiary, etc, of the form $\chi_{Z}\left(q^{a}, p^{a}\right)=0$ with $Z=1 \ldots A$ with $A$ the total number of independent constraints, that are preserved under the evolution given by the equations (2425) provided part or all the arbitrary functions $V$ are conveniently fixed.

As in the continuum case it is convenient to introduce the notion of first and second class constraints, in order to quantize the theory. A constraint is of first class if it commutes with all the constraints, if that is not the case it is of second class. As in the continuum case one can define first class functions of the canonical variables $f(q, p)$ that are not necessarily constraints. Such a function will be first class if it commutes with all the constraints. Second class constraints may be imposed strongly by introducing Dirac brackets. As the evolution equations preserve the Poisson structure, they will preserve de Dirac structure because Dirac brackets are defined in terms of Poisson brackets. One ends up with a theory with a set of evolution equations that preserve the symplectic structure of the system, and therefore may be quantized by describing the evolution in terms of unitary operators.

In the discrete case there is not a straightforward relation between the number of first and second class constraints and the number of phase space degrees of freedom. This is due to the fact that now the evolution of the constraints is not directly related with their Poisson brackets with a total Hamiltonian. Thus, the fact that a constraint does not commute with others is not easily related with the determination of an arbitrary function. It is still very easy to determine the number of phase space degrees of freedom. In fact this number is given by two times the number of configuration variables minus the total number of constraints minus the number of arbitrary functions $v$.

\section{AN EXAMPLE OF CONSTRAINED SYSTEM WITH SECOND CLASS CONSTRAINTS}

To illustrate the techniques elaborated above, we would like to discuss a model that is simple, yet addresses in a non-trivial way the main points we discussed. This example had been treated using ad-hoc techniques in [4]. The model consists of a parameterized free particle in a two dimensional space-time under the influence of a linear potential. The discrete Lagrangian is given by,

$$
L_{n} \equiv L\left(q_{n}^{a}, \pi_{n}^{a}, N_{n}, q_{n+1}^{a}, \pi_{n+1}^{a}, N_{n+1}\right)=\pi_{n}^{a}\left(q_{n+1}^{a}-q_{n}^{a}\right)-N_{n}\left[\pi_{n}^{0}+\frac{1}{2}\left(\pi_{n}^{1}\right)^{2}+\alpha q_{n}^{1}\right] .
$$

We have chosen a first order formulation for the particle. However, this Lagrangian is of the type we considered in this paper, one simply needs to consider all variables, $q^{a}, \pi^{a}, N$ as configuration variables. The system is clearly singular since the $\pi^{\prime} s$ and $N$ only appear at level $n$ (or in the continuum Lagrangian, their time derivatives are absent). When 
considered as a Type I generating function, the above Lagrangian leads to the equations

$$
\begin{aligned}
p_{\pi, n+1}^{a} & =\frac{\partial L_{n}}{\partial \pi_{n+1}^{a}}=0 \\
p_{q, n+1}^{a} & =\frac{\partial L_{n}}{\partial q_{n+1}^{a}}=\pi_{n}^{a} \\
p_{N, n+1} & =\frac{\partial L_{n}}{\partial N_{n+1}}=0
\end{aligned}
$$

and

$$
\begin{aligned}
& p_{\pi, n}^{a}=-\frac{\partial L_{n}}{\partial \pi_{n}^{a}}=-\left(q_{n+1}^{a}-q_{n}^{a}\right)+\pi_{n}^{1} N_{n} \delta_{1}^{a}+N_{n} \delta_{0}^{a}, \\
& p_{q, n}^{a}=-\frac{\partial L_{n}}{\partial q_{n}^{a}}=\pi_{n}^{a}+\delta_{1}^{a} \alpha N_{n}, \\
& p_{N, n}=-\frac{\partial L_{n}}{\partial N_{n}}=\pi_{n}^{0}+\frac{1}{2}\left(\pi_{n}^{1}\right)^{2}+\alpha q_{n}^{1} .
\end{aligned}
$$

One can easily recognize that the system has six constraints: three at the $n+1$ level, and three at the $n$ level. They are:

$$
\begin{aligned}
\psi_{1}^{a} & \equiv p_{\pi, n+1}^{a}=0 \\
\psi_{2} & \equiv p_{N, n+1}=0 \\
\Phi_{1}^{a} & \equiv p_{q, n}^{a}-\left(\pi_{n}^{a}+\delta_{1}^{a} \alpha N_{n}\right), \\
\Phi_{2} & \equiv p_{N, n}-\left[\pi_{n}^{0}+\frac{1}{2}\left(\pi_{n}^{1}\right)^{2}+\alpha q_{n}^{1}\right] .
\end{aligned}
$$

Therefore the evolution depends on three arbitrary functions $V_{N, n}, V_{\pi, n}^{a}$,

$$
\begin{aligned}
q_{n+1}^{a} & =q_{n}^{a}+\pi_{n}^{1} N_{n} \delta_{1}^{a}+N_{n} \delta_{0}^{a}-p_{\pi, n}^{a} \\
\pi_{n+1}^{a} & =\pi_{n}^{a}+V_{\pi, n}^{a} \\
N_{n+1} & =N_{n}+V_{N, n} .
\end{aligned}
$$

The preservation of the $\psi$ constraints from level $n$ to level $n+1$ is automatically ensured from (23). Now we impose the preservation of the $\Phi$ constraints upon evolution. Let us begin with $\Phi_{1}^{0}$ :

$$
\Phi_{1 n+1}^{0} \equiv p_{q, n+1}^{0}-\pi_{n+1}^{0}=p_{q, n}^{0}-\pi_{n}^{0}-V_{\pi, n}^{0}=0,
$$

which taking into account the constraint $\Phi_{1 n}^{0}$ implies $V_{\pi, n}^{0}=0$.

For the $\Phi_{2}$ one gets the equation

$$
\Phi_{2 n+1}=\pi_{n}^{0}+\alpha\left(q_{n}^{1}+\pi_{n}^{1} N_{n}\right)+\left(\pi_{n}^{1}+V_{n}^{\pi 1}\right)^{2} / 2=0,
$$

that taking into account the constraint $\Phi_{2 n}$ implies that

$$
V_{\pi, n}^{1}=-\pi_{n}^{1}+\epsilon \sqrt{\left(\pi_{n}^{1}\right)^{2}-2 \pi_{n}^{1} N_{n} \alpha}
$$

where $\epsilon= \pm 1$.

Finally we have

$$
\Phi_{1 n+1}^{1}=p_{q, n}^{1}-N_{n} \alpha-\pi_{n}^{1}-V_{\pi, n}^{1}-\alpha\left(N_{n}+V_{N, n}\right)=0,
$$

that after imposing the constraint at level $n$ leads to

$$
V_{N, n}=-\frac{1}{\alpha} V_{\pi, n}^{1}-N_{n} .
$$


Thus, the evolution equations for the configuration variables are

$$
\begin{aligned}
q_{n+1}^{a} & =q_{n}^{a}+\pi_{n}^{1} N_{n} \delta_{1}^{a}+N_{n} \delta_{0}^{a} \\
\pi_{n+1}^{0} & =\pi_{n}^{0}, \\
\pi_{n+1}^{1} & =\epsilon \sqrt{\left(\pi_{n}^{1}\right)^{2}-2 \pi_{n}^{1} N_{n} \alpha}, \\
N_{n+1} & =\frac{1}{\alpha}\left[\pi_{n}^{1}-\epsilon \sqrt{\left(\pi_{n}^{1}\right)^{2}-2 \pi_{n}^{1} N_{n} \alpha}\right] .
\end{aligned}
$$

We are now ready to define an invertible canonical transformation with the help of a type 3 generating function. Notice that these evolution equations are invertible for $q_{n}^{a}$, and therefore we are in the case I. The inverse is given by a set of equations of the form $q_{n}\left(q_{n+1}\right)$, explicitly given by,

$$
\begin{aligned}
q_{n}^{1} & =q_{n+1}^{1}-\pi_{n+1}^{1} N_{n+1}-\frac{\alpha}{2}\left(N_{n+1}\right)^{2} \\
q_{n}^{0} & =q_{n+1}^{0}-\frac{\pi_{n+1}^{1} N_{n+1}+\frac{\alpha}{2}\left(N_{n+1}\right)^{2}}{\pi_{n+1}^{1}+\alpha N_{n+1}} \\
\pi_{n}^{0} & =\pi_{n+1}^{0} \\
\pi_{n}^{1} & =\pi_{n+1}^{1}+\alpha N_{n+1} \\
N_{n} & =\frac{\pi_{n+1}^{1} N_{n+1}+\frac{\alpha}{2}\left(N_{n+1}\right)^{2}}{\pi_{n+1}^{1}+\alpha N_{n+1}} .
\end{aligned}
$$

Recalling that $F_{3}$ is given by equation (28) we obtain,

$$
\begin{aligned}
F_{3}= & p_{\pi, n}^{0} \pi_{n+1}^{0}+p_{\pi, n}^{1}\left(\alpha N_{n+1}+\pi_{n+1}^{1}\right)+p_{q, n}^{0} q_{n+1}^{0}+\frac{1}{2} N_{n+1}\left[\alpha^{2} N_{n+1}^{2}+3 \alpha N_{n+1} \pi_{n+1}^{1}+2\left(\pi_{n+1}^{1}\right)^{2}\right] \\
& -\frac{N_{n+1}\left(\alpha N_{n+1}+2 \pi_{n+1}^{1}\right)}{2\left(\alpha N_{n+1}+\pi_{n+1}^{1}\right)}\left[p_{q, n}^{0}-p_{N, n}+\frac{1}{2}\left(\pi_{n+1}^{1}\right)^{2}+\alpha q_{n+1}^{1}\right]+p_{q, n}^{1}\left(q_{n+1}^{1}-\frac{\alpha}{2} N_{n+1}^{2}-N_{n+1} \pi_{n+1}^{1}\right) .
\end{aligned}
$$

One can check that Eqs. (61) to 655) are easily recovered by taking the partial derivative with respect to $p_{n}^{a}$. By differentiating with respect to $q_{n+1}^{a}$ one gets

$$
\begin{aligned}
p_{q, n+1}^{0}= & p_{q, n}^{0} \\
p_{q, n+1}^{1}= & p_{q, n}^{1}-\frac{\alpha}{2} A_{n} N_{n+1}\left(\alpha N_{n+1}+2 \pi_{n+1}^{1}\right), \\
p_{\pi, n+1}^{0}= & p_{\pi, n}^{0} \\
p_{\pi, n+1}^{1}= & p_{\pi, n}^{1}+\frac{N_{n+1}}{2}\left[3 \alpha N_{n+1}-2 p_{q, n}^{1}+4 \pi_{n+1}^{1}+2 B_{n} A_{n}-A_{n}\left(\pi_{n+1}^{1}+B_{n} A_{n}\right)\left(\alpha N_{n+1}+2 \pi_{n+1}^{1}\right)\right], \\
p_{N, n+1}= & \frac{3}{2} \alpha^{2} N_{n+1}^{2}+\alpha p_{\pi, n}^{1}-p_{q, n}^{1} \pi_{n+1}^{1}+\left(\pi_{n+1}^{1}\right)^{2}+B_{n} \\
& +\alpha N_{n+1}\left[-p_{q, n}^{1}+3 \pi_{n+1}^{1}-\frac{1}{2} B_{n} A_{n}^{2}\left(\alpha N_{n+1}+2 \pi_{n+1}^{1}\right)\right]
\end{aligned}
$$

where we have introduced

$$
\begin{aligned}
& A_{n} \equiv\left(\alpha N_{n+1}+\pi_{n+1}^{1}\right)^{-1}, \\
& B_{n} \equiv p_{N, n}-p_{q, n}^{0}-\frac{1}{2}\left(\pi_{n+1}^{1}\right)^{2}-\alpha q_{n+1}^{1} .
\end{aligned}
$$

By substituting in Eqs.(67) to (71) the variables $q_{n+1}$ and using the level $n$ constraints one gets Eqs. (39) to (41), which is a canonical transformation that reproduces, on the constraint surface, the evolution equations of the discrete particle.

What remains to be done is to identify the second class constraints and impose them strongly. The complete set of six constraints of this model $\psi$ and $\Phi$ are second class and allow to solve for $\pi^{a}$ and $N$ and eliminate completely these variables and their complex conjugates $P_{\pi}$ and $P_{N}$. 
One can proceed in two different ways. The first alternative is to start by observing that $P_{\pi}$ and $P_{N}$ vanish strongly, and then to solve for $\pi$ and $N$ in terms of the $n+1$ level variables, leading to

$$
\begin{aligned}
\pi_{n}^{0} & =p_{q, n+1}^{0}, \\
\pi_{n}^{1} & =p_{q, n+1}^{1}, \\
N_{n} & =\frac{C_{n+1}}{\alpha p_{q, n+1}^{1}},
\end{aligned}
$$

where $C_{n+1}=p_{q, n+1}^{0}+\left(p_{q, n+1}^{1}\right)^{2} / 2+\alpha q_{n+1}^{1}$. The relevant evolution equations are obtained from (5766768), and are given by

$$
\begin{aligned}
& q_{n+1}^{0}=q_{n}^{0}+N_{n}=q_{n}^{0}+\frac{C_{n+1}}{\alpha p_{q, n+1}^{1}} \\
& q_{n+1}^{1}=q_{n}^{1}+N_{n} \pi_{n}^{1}=q_{n}^{1}+\frac{C_{n+1}}{\alpha} \\
& p_{q, n}^{0}=p_{q, n+1}^{0}, \\
& p_{q, n}^{1}=p_{q, n+1}^{1}+\alpha N_{n}=p_{q, n+1}^{1}+\frac{C_{n+1}}{p_{q, n+1}^{1}}
\end{aligned}
$$

and we recover the evolution equations obtained in [4].

The second alternative consists in solving for $\pi$ and $N$ in terms of the $n$ level variables, leading to

$$
\begin{aligned}
\pi_{n}^{0} & =p_{q, n}^{0}, \\
\pi_{n}^{1} & =\epsilon \sqrt{-2\left(p_{q, n}^{0}+\alpha q_{n}^{1}\right)}, \\
N_{n} & =\frac{1}{\alpha}\left(p_{q, n}^{1}-\epsilon \sqrt{-2\left(p_{q, n}^{0}+\alpha q_{n}^{1}\right)}\right),
\end{aligned}
$$

and from here, computing the evolution equations by using (57/67]68). The two methods yield evolution schemes of different functional form since one propagates "forward" in time and the other "backward". The inequivalence in the functional form stems from the fact that the discretization of the time derivatives chosen in the Lagrangian is not centered. It should be emphasized that if one starts from given initial data and propagates forwards with the first system of equations and then backwards using the second, one will return to the same initial data.

Notice that we have 6 second class constraints, and the initial number of phase space degrees of freedom was 10 . By noticing that there are no arbitrary functions left, one is left with 4 degrees of freedom on the constraint surface. The continuum model had two degrees of freedom.

The procedure we have followed here is completely general and may be simplified when one is treating specific cases. For instance, as it happens in the continuum theory [8] it is sometimes possible to implement the canonical analysis by first solving the constraints for the unphysical degrees of freedom $N, \pi, p^{N}, p^{\pi}$ and then introducing a generating functional on the physical degrees of freedom by following the procedure of the previous sections. In this particular case it is easy to show that, for $p^{q^{1}} \neq 0,\left|\frac{\partial q_{n+1}^{a}}{\partial q_{n}^{b}}\right| \neq 0$, where $q^{a} \equiv\left(q^{1}, q^{0}\right)$, and thus it is possible to construct an $F_{3}$ generating functional. In all the models treated up to now in the literature the unphysical degrees of freedom were eliminated before obtaining the canonical transformation for the evolution of the physical degrees of freedom. In order to keep the analysis general in a simple model, here we have kept all the variables involved in this approach.

\section{TREATMENT IN TERMS OF TYPE II GENERATING FUNCTIONS}

Up to now, we have taken as the starting point a singular Type I generating function given by $F_{1}=-L\left(q_{n}^{a}, q_{n+1}^{a}\right)$. It is interesting to analyze how singular systems may be described in terms of other types of generating functions.

Let us assume that neither condition (8) nor condition (5) are fulfilled by the discrete system and, therefore, we are in the singular case. Then, it may be immediately seen, by using (16), and taking into account that the determinant that appears in (8) now vanishes, that singular systems with first order Lagrangians in the continuum lead to the presence of pseudo-constraints $\phi_{\alpha}\left(q_{n}, p_{n+1}\right)=0$, in the theory. Recall that these pseudo-constraints are similar to the constraints that arise in the continuum case, but mixing configuration variables at one level with momenta in the 
next level. As in the continuum case, one can then introduce the pseudo-constraint surface $S_{\phi}$ which we shall assume has well defined functions,

$$
\widetilde{\nabla} \phi_{\alpha}=\left(\ldots \frac{\partial \phi}{\partial q_{n}^{a}} \ldots, \ldots \frac{\partial \phi}{\partial p_{n+1}^{a}} \ldots\right)
$$

where a vector $\tilde{\tau}$ in the tangent space of $S_{\phi}, T_{(q, p)} S_{\phi}$, is such that

$$
\tilde{\tau} \cdot \widetilde{\nabla} \phi_{\alpha}=0 \quad \forall \alpha .
$$

Let us introduce now as before the type-2 Hamiltonian (12),

$$
H_{2} \equiv \sum_{a} p_{n+1}^{a}\left(q_{n+1}^{a}-q_{n}^{a}\right)-L\left(q_{n}, q_{n+1}\right),
$$

For which it is easy to see, using the pseudo-constraints, that it is a function of $q_{n}, p_{n+1}$. Let us consider now an infinitesimal variation of $\mathrm{H}_{2}$ along the pseudo-constraint surface,

$$
\begin{aligned}
d H_{2} & =\sum_{a}\left[\left(q_{n+1}^{a}-q_{n}^{a}\right) d p_{n+1}^{a}+\left(p_{n+1}^{a}-\frac{\partial L}{\partial q_{n+1}^{a}}\right) d q_{n+1}^{a}-\left(p_{n+1}^{a}+\frac{\partial L}{\partial q_{n}^{a}}\right) d q_{n}^{a}\right] \\
& =\sum_{a}\left[\left(q_{n+1}^{a}-q_{n}^{a}\right) d p_{n+1}^{a}-\left(p_{n+1}^{a}+\frac{\partial L}{\partial q_{n}^{a}}\right) d q_{n}^{a}\right]
\end{aligned}
$$

where in the last step we used ([6). $H_{2}$ is well defined in $S_{\phi}$ but can be extended to the whole phase space as it is done in the case of constraints in the continuum theory. In order to obtain the canonical equation of motion we start from the identity,

$$
d H_{2}\left(q_{n}, p_{n+1}\right)=\sum_{a}\left(\frac{\partial H_{2}}{\partial q_{n}^{a}} d q_{n}^{a}+\frac{\partial H_{2}}{\partial p_{n+1}^{a}} d p_{n+1}^{a}\right)
$$

and evaluating it for an infinitesimal displacement $(d q, d p)$ in $T_{(q, p)} S_{\phi}$, we can use (87) and the Lagrangian equations of motion (7) to obtain,

$$
\sum_{a}\left[\left(p_{n+1}^{a}-p_{n}^{a}+\frac{\partial H_{2}}{\partial q_{n}^{a}}\right) d q_{n}^{a}+\left(q_{n}^{a}-q_{n+1}^{a}+\frac{\partial H_{2}}{\partial p_{n+1}^{a}}\right) d p_{n+1}^{a}\right]=0 .
$$

Since $(d q, d p)$ is an arbitrary tangent vector to $S_{\phi}$, following (85) we obtain that the coefficient must be proportional to the gradient. Introducing therefore the Lagrange multipliers $\lambda^{a}$ as the proportionality factors, we will end with the set of equations,

$$
\begin{aligned}
q_{n+1}^{a} & =q_{n}^{a}+\frac{\partial H_{2}}{\partial p_{n+1}^{a}}+\lambda_{n}^{\alpha} \frac{\partial \phi_{\alpha}}{\partial p_{n+1}^{a}}, \\
p_{n+1}^{a} & =p_{n}^{a}-\frac{\partial H_{2}}{\partial q_{n}^{a}}-\lambda_{n}^{\alpha} \frac{\partial \phi_{\alpha}}{\partial q_{n}^{a}} \\
\phi_{\alpha}\left(q_{n}, p_{n+1}\right) & =0 .
\end{aligned}
$$

This system is similar to the continuum set of equations with the inclusion of pseudo-constraints, which have the same functional form as the continuum constraints, but involve configuration variables at level $n$ and momenta at level $n+1$, instead of $n$ level variables. From this initial evolution equations one may follow a procedure similar to the one developed in the previous section in order to study the consistency of the (primary) constraints and pseudo-constraints.

\section{DISCUSSION AND CONCLUSIONS}

We have provided a canonical procedure for the introduction of a preserved symplectic structure in discrete constrained systems. The analogy with Dirac's procedure in the continuum is quite remarkable. It is possible to define a notion of discrete evolution that weakly preserves constraints and Poisson brackets. The distinction between first 
and second class constraints is still useful and when second class constraints are imposed strongly the resulting Dirac brackets are preserved.

A feature of the discretized theories is that they may have a smaller number of first class constraints, and consequently more degrees of freedom than the continuum counterparts. The extra degrees of freedom come from the fact that the discrete theories may not necessarily have the same symmetries as the continuum theories. For instance, in the case of homogeneous cosmologies studied in [9] the extra pair of phase space degrees of freedom are associated with the fact that in the discrete theory different choices of refinements in the discretization in time correspond to different solutions in the discrete theory that nevertheless approximate the same solution in the continuum theory.

This is only a first step for a complete understanding of the dynamics of discrete gauge systems. The relation between the number of constraints of first and second class and the number of degrees of freedom, and the connection between the first class constraints and the gauge invariance of the discrete dynamical system need to be further studied. Moreover, as discussed in the body of the article, if one wishes to consider more pathological systems than the ones considered here, more elaborate canonical transformations may need to be introduced.

The issue of the continuum limit is well understood in the non singular case, where there is an external step parameter that controls the approximation. However, it needs further study in the case of singular systems, particularly in the case of totally constrained systems where the step of the approximation is encoded in the additional degrees of freedom of the discrete theory. This issue has been studied in several models [4, 9] but a complete characterization of the possible behaviors is still lacking. A similar comment applies to the role of spatial discretizations when one is considering lattice field theories.

\section{ACKNOWLEDGMENTS}

This paper was motivated by a series of questions by Karel Kuchař about our previous work. This work was supported by grant nsf-phy0244335 and funds from the Horace Hearne Jr. Laboratory for Theoretical Physics and the Abdus Salam International Center for Theoretical Physics.

[1] C. Di Bartolo, R. Gambini, J. Pullin, Class. Quan. Grav. 19, 5475 (2002).

[2] R. Gambini and J. Pullin, Phys. Rev. Lett. 90, 021301, (2003).

[3] J. Marsden, M. West, Acta Numerica 10, 357 (2001).

[4] R. Gambini, R.A. Porto and J. Pullin, in "Recent developments in gravity", K. Kokkotas, N. Stergioulas, World Scientific, Singapore, (2003) arXiv:gr-qc/0302064.

[5] R. Gambini, R. A. Porto and J. Pullin, New Journal of Physics 6, 45 (2004).

[6] R. Gambini, R. A. Porto and J. Pullin, Class. Quan. Grav. 21 L51 (2004).

[7] P. A. M. Dirac, "Lectures on Quantum Mechanics", Dover (2001)

[8] M. Henneaux and C. Teitelboim, "Quantization of Gauge Systems", Princeton University Press (1994).

[9] R. Gambini and J. Pullin, Class. Quan. Grav. 20, 3341 (2003). 